\title{
Exploring the Stability of Twisted van der Waals Heterostructures
}

\author{
Andrea Silva, ${ }^{*,+,}$ Victor E.P. Claerbout, ${ }^{*, \Phi}$ Tomas Polcar, ${ }^{\dagger,+, \mathbb{q}}$ Denis Kramer, ${ }^{\dagger, \xi}$ \\ and Paolo Nicolini \\ $\dagger$ Engineering Materials, University of Southampton \\ $\ddagger$ national Centre for Advanced Tribology Study at University of Southampton \\ \Advanced Materials Group, Department of Control Engineering, Faculty of Electrical \\ Engineering, Czech Technical University in Prague (CTU) \\ $\S$ Mechanical Engineering, Helmut Schmidt University, Hamburg, Germany \\ E-mail: a.silva@soton.ac.uk; claervic@fel.cvut.cz
}

\begin{abstract}
Recent research showed that the rotational degree of freedom in stacking 2D materials yields great changes in the electronic properties. Here, we focus on an often overlooked question: are twisted geometries stable and what defines their rotational energy landscape? Our simulations show how epitaxy theory breaks down in these systems, and we explain the observed behaviour in terms of an interplay between flexural phonons and the interlayer coupling, governed by moiré superlattice. Our argument, applied to the well-studied $\mathrm{MoS}_{2} /$ Graphene system, rationalizes experimental results and could serve as guidance to design twistronics devices.
\end{abstract}




\section{Keywords}

Heterostructures, Molybdenum Disulfide, Graphene, Twist Deformation, Molecular Dynamics Simulations, Twistronics

\section{Introduction}

Van der Waals (vdW) 2D materials, like graphite and the family of transition metal dichalcogenides (TMDs), are a class of compounds characterized by an anisotropic structure. Strong intralayer covalent bonds complement the weak vdW interlayer interactions, which facilitate the lamellar structure of bulk crystals. Due to their diverse chemistry and versatile properties, these materials have received significant attention from the scientific community in

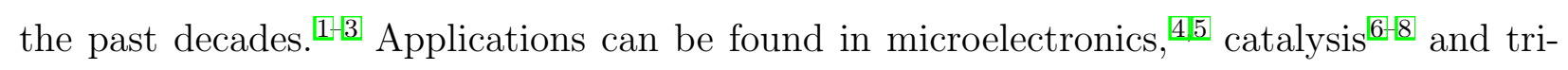
bology. 9110 While the attractive properties of the pure compounds are widely known, recent efforts have been focusing on the physics and properties emerging from the stacking degree of freedom offered by these lamellar materials. Different types of single layers can be mixed and matched to create new superstructures, termed heterostructures.11140 A key feature, affecting the behaviour of multi-layered structures, is the relative orientational mismatch between layers. While heterostructures are intrinsically incommensurate due to the different lattice constants of the parent single layers, incommensurability can also arise in homostructrures due to a relative misalignment of the single layers. ${ }^{9}$

The relative mismatch between layers, both for homo- and heterostructures, has been related to a range of electronic and mechanical properties. $\frac{915]}{20}$ A flourishing new branch in the field of condensed matter, known as twistronics, promises to allow fine-tuning of the electronic properties using the rotational misalignment between layers. $\frac{1721}{121}$ A notable example is the recent discovery of unconventional superconductivity in bilayer graphene $(G)$ twisted at the magic angle of $1.1^{\circ}$.21 Another study found that the vertical conductivity of bilayer $\mathrm{MoS}_{2} / \mathrm{G}$ heterostructures varies by a factor of five when imposing an angle of $30^{\circ}$ between 
the layers. ${ }^{222}$ Finally, a pioneering work ${ }^{92}$ showed that, by switching from commensurate to incommensurate orientation in graphite systems, it is possible to achieve a state in which the coefficient of friction vanishes, the so-called superlubric regime.

Despite the interesting physics, that results from these relative mismatches, an often overlooked question is by what their rotational energy landscape is determined, and thus which geometries are stable. Indeed, experimental studies are contradictory on this point, with a wide range of misfit angles found, even for the same type of system. $23 \sqrt[26]{26}$ Below, we give a few examples of heterostructures based on $\mathrm{MoS}_{2}$ on G. This system may be regarded as the prototypical 2D heterostructure, as it combines two well-known and extensively studied materials, widely reported on in the literature. Moreover, it has been reported that $\mathrm{MoS}_{2} / \mathrm{G}$ heterostructures show interesting electronic behaviour as function of their mismatch angle. ${ }^{27128}$ Using chemical vapor deposition (CVD), Liu et al. epitaxially grew triangles of $\mathrm{MoS}_{2}$ on top of $\mathrm{G}$, about $0.135 \mu \mathrm{m}$ in size, with the majority of them (84\%) aligned to the substrate and the remainder rotated by $30^{\circ} .{ }^{23}$ Using the same technique, Shi et al. found mismatch angles between $\mathrm{MoS}_{2}$ and $\mathrm{G}$, on top of a $\mathrm{Cu}$ foil, ranging from $-11^{\circ}$ to $18^{\circ}$, with hexagonal flake sizes of about $1 \mu \mathrm{m} \cdot{ }^{[24}$ For CVD-grown flakes of $9 \mu \mathrm{m}, \mathrm{Lu}$ et al. found a mismatch with typical angles below $\left.3^{\circ} .25\right]$ Finally, using an exfoliation protocol, Adrian et al. prepared multi-layered heterostructures and observed a misfit angle of $7.3^{\circ} .26$ As well as different values for the observed mismatch angle, these studies offer different explanations for its origin. Whereas some attribute the observed (mis)alignment to the vdW epitaxy, accommodating the mismatch in lattice constants, $\stackrel{2324}{224}$ others use the structural features of the underlying $\mathrm{G}$ and the edges ${ }^{\sqrt{25}}$ as an explanation.

In a recent theoretical work, Zhu et al. .155 explained the orientational ordering of finite size homostructures, e.g. $\mathrm{MoS}_{2}$ flakes on an $\mathrm{MoS}_{2}$ substrate, using a purely geometrical argument: the lowest energy configuration is the one obtained by the roto-translation of the rigid flake which maximizes the number of locally commensurate regions. Although this argument is solely based on geometry, it provides a satisfactory approximation for finite size 
systems but in the limit of infinite planes, i.e. for large enough flakes, commensurate regions equal incommensurate ones. Therefore, in the limit of extended interfaces, other theoretical frameworks are needed.

In this contribution, we aim to explore the energy landscape originating from the rotational degree of freedom of edge-free, complex layered heterostructures and relate its fundamental origin to incommensurability and layer deformation at imposed angles. This will provide guidance for the design of $\mathrm{vdW}$ heterostructures and the control of the twisting degree of freedom. In order to make a more general point about the relative importance of different contributions, in determining the total energy landscape, we focus on a specific but well-studied system, namely $\mathrm{MoS}_{2} / \mathrm{G}$. While previous energy analysis focused on commensurate $\mathrm{MoS}_{2} / \mathrm{MoS}_{2}$ homostructures ${ }^{29}$ or near-commensurate 2D-crystal G/hBN heterostructures,, 30 the $\mathrm{MoS}_{2} / \mathrm{G}$ BL system has a mismatch ratio $\rho=l / l \approx 0.8$ far away from commensurate value $\rho=1$ and moreover is composed of a mixture of $2 \mathrm{D}$ and 3D crystal monolayers. This analysis shows the practical application of our argument and will also allow us to comment on the apparently contradictory experimental observations regarding this particular system.

\section{Results and discussion}

In order to avoid finite-size effects and harvest information solely from the relaxation of the atoms in the layers, we implemented a protocol to build edge-free geometries. The resulting supercells are simultaneously compatible with the lattice mismatch and a relative imposed angle between the layers. As a result, periodic boundary conditions (PBC) can be applied to these structures. The starting interface geometry is described by a pair of $2 \mathrm{D}$ lattices defined by vectors $\left(l_{\mathrm{a}} \hat{\mathbf{a}}_{1}, l_{\mathrm{a}} \hat{\mathbf{a}}_{2}\right)$ and $\left(l_{\mathrm{b}} \hat{\mathbf{b}}_{1}, l_{\mathrm{b}} \hat{\mathbf{b}}_{2}\right)$, where $l_{\mathrm{a}}$ and $l_{\mathrm{b}}$ represent the lattice constants, and the $\hat{\mathbf{b}}_{i}$ vectors are rotated by an angle $\theta$ with respect to $\hat{\mathbf{a}}_{i}$. Two layers will be compatible if they satisfy the matching condition $l_{\mathrm{a}}\left(n_{1} \hat{\mathbf{a}}_{1}+n_{2} \hat{\mathbf{a}}_{2}\right)=l_{\mathrm{b}}\left(m_{1} \hat{\mathbf{b}}_{1}+m_{2} \hat{\mathbf{b}}_{2}\right)$, where the integer 
numbers $n_{1}, n_{2}, m_{1}, m_{2}$ are supercell indices representing the repetition along each lattice vector. In practice, for incommensurate lattice constants, the matching condition yielding PBC-compliant supercells can only be satisfied approximately, i.e. the lattice spacing $l^{\prime}$ of one of the two component needs to deviate from its equilibrium value $l$. Here, in order to obtain suitable structures with imposed angles between $0^{\circ}$ and $60^{\circ}$, we accept supercells satisfying $\left|l^{\prime}-l\right|<5 \times 10^{-7} \AA$. The resulting strain is applied to the $\mathrm{MoS}_{2}$ layer, which leads to a maximum strain $\epsilon=\frac{l^{\prime}-l}{l}$ within the same order of magnitude, four orders less than reported strains in other computational studies. ${ }^{31}[33$ This protocol yields a set of supercells, each of which has a different number of atoms up to 343893, created according to the four supercell indices resulting in an unique twisting angle, satisfying the matching condition. Details of this protocol and all the parameters of the supercells used are reported in the Supplementary Information (SI).

In these supercells, we distinguish intralayer and interlayer inter-atomic interactions, resulting in the following Hamiltonian

$$
H=H_{\mathrm{L}_{1}}+H_{\mathrm{L}_{2}}+H_{\mathrm{L}_{1} \mathrm{~L}_{2}} .
$$

The $\mathrm{G}$ layer is modelled with the REBO potential, ${ }^{34} H_{\mathrm{L}_{1}}=H_{\mathrm{C}}^{(\mathrm{REBO})}$, while the 3-body Stillinger-Weber (SW) potential ${ }^{333}$ is used for $\mathrm{MoS}_{2}, H_{\mathrm{L}_{2}}=H_{\mathrm{MoS}_{2}}^{(\mathrm{SW})}$. Interlayer coupling is described by the Lennard-Jones (LJ) potential

$$
\begin{aligned}
H_{\mathrm{L}_{1} \mathrm{~L}_{2}} & =H_{\mathrm{C}-\mathrm{Mo}, \mathrm{C}-\mathrm{S}}^{(\mathrm{LJ})} \\
& =\sum_{\substack{i \in C \\
j \in M o, S}} 4 \epsilon_{i j}\left[\left(\frac{\sigma_{i j}}{r}\right)^{12}-\left(\frac{\sigma_{i j}}{r}\right)^{6}\right] .
\end{aligned}
$$

Since the interlayer interactions are especially relevant for the aim of this work, we refined the values for the C-Mo and C-S interactions found in Ref. ${ }^{[3]}$ As a reference set, we computed the binding energy curves at DFT level, using the Vienna Ab initio Simulation Pack- 
age (VASP) ${ }^{\sqrt[35136]{16}}$ within the Projector Augmented-Wave (PAW) framework. ${ }^{[37}$ The exchangecorrelation potential is approximated using the PBE functional ${ }^{[38}$ and the vdW dispersion is described by the DFT-D2 method. ${ }^{[39}$ After this procedure, we are able to perform energy minimizations using the conjugate gradient algorithm available within the LAMMPS package. ${ }^{40]}$ More details about the fitting and minimization procedure can be found in the SI. An overview of the computational setup can be found in Figure 1 .

An approximate theory for the orientational ordering of an incommensurate interface was proposed by Novaco and McTague. $\frac{4142}{}$ Although developed in the context of epitaxial growth of noble gas layers on metal surfaces, it has been successfully applied to the behaviour of mesoscopic colloidal layers in optical lattices ${ }^{43}$ and metal clusters adsorbed on G. ${ }^{44}$ The assumption of the Novaco-McTague (NM) theory is that two purely 2D systems, linked via an interface, may be divided into two separate components: a soft adsorbate layer, treated within the harmonic approximation, atop a rigid substrate. This means that one of the intralayer terms in Eq. (11) is substituted by its harmonic approximation, while the coordinates of the second layer are frozen at its initial values, $r_{0}$. For example, considering $\mathrm{G}$ as the adsorbate and $\mathrm{MoS}_{2}$ as the substrate yields the following Hamiltonian

$$
H_{\mathrm{NM}}=\left.H_{\mathrm{C}}^{(\mathrm{REBO})}\right|_{\text {harmonic }}+\left.H_{\mathrm{MoS}_{2}}^{(\mathrm{SW})}\right|_{r_{0}}+H_{\mathrm{C}-\mathrm{MO}, \mathrm{C}-\mathrm{S}} .
$$

If the substrate and the adsorbate lattices are incommensurate, due to a mismatch in lattice constants, the system is frustrated: the intralayer interactions within the adsorbate favor the intrinsic equilibrium lattice spacing, while the interactions with the substrate drive the atoms away from their equilibrium positions. In the limit of long wavelength distortions, the NM theory predicts that the system can lower its energy by converting part of the longitudinal stress coming from the incommensurability into shear stress. This yields a 


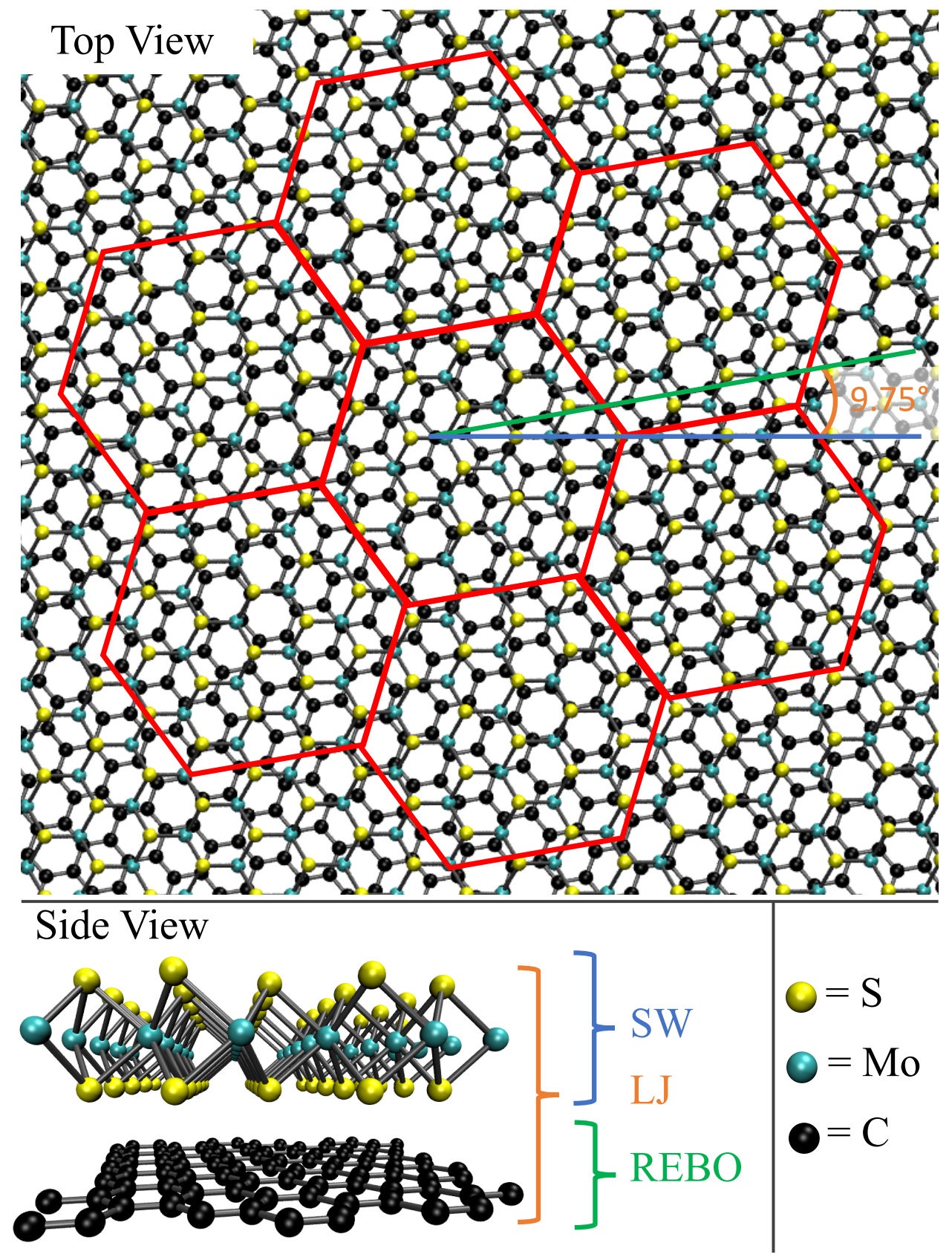

Figure 1: Schematic overview of the computational setup displayed for a mismatch angle of $9.75^{\circ}$. In the top panel, a top view is provided, including a sketch of moiré tiling resulting from the geometrical interference between the two lattices. The mismatch angle between the lattice direction of $\mathrm{MoS}_{2}$ (blue line) and $\mathrm{G}$ (green line) lattices is reported as well. In the bottom panel, a side view is provided, including the corresponding force fields that have been used. 
small misalignment angle between the two lattices given by

$$
\cos \theta_{\mathrm{NM}}=\frac{1+\rho^{2}(1+2 \delta)}{\rho\left[2+\delta\left(1+\rho^{2}\right)\right]},
$$

where $\rho=l_{\text {substrate }} / l_{\text {adsorbate }}$ is the mismatch ratio between the two lattices and $\delta=\left(c_{\mathrm{L}} / c_{\mathrm{T}}\right)^{2}-$ 1, with $c_{\mathrm{T}}$ and $c_{\mathrm{L}}$ being the transverse and longitudinal sound velocities of the adsorbate, respectively.

The result of NM in Eq. (4) can be applied to our system by extracting the sound velocity of each single layer from the phonon dispersion, as reported in the SI. There are two possible scenarios: $\mathrm{G}$ can be treated as the rigid substrate, while $\mathrm{MoS}_{2}$ acts as a soft adsorbate, or vice versa. In the first case, the theory predicts $\theta_{\mathrm{NM}}^{\mathrm{MoS}_{2}}=8.0^{\circ}$, while if $\mathrm{G}$ is the adsorbate, the minimum-energy angle is $\theta_{\mathrm{NM}}^{\mathrm{G}}=8.6^{\circ}$. The prediction of the NM model can be verified by minimizing the total energy of the twisted geometries described above under suitable constraints. We froze the atoms of the heterostructures in the direction perpendicular to the surface, i.e. the $z$ axis, effectively reducing the dimensionality of the system to $2 \mathrm{D}$. Furthermore, we also froze the atoms of the substrate layer in the in-plane directions $x$ and $y$, enforcing a fully rigid substrate.

As mentioned at the beginning of this section and explained in detail in Section one of the SI, the edge-free geometries used in this work inevitably retain a degree of stress resulting from the matching condition for the two lattices in order to be able to apply $\mathrm{PBC}$. The slightly different strains, unique to every geometry, result in different offsets in the obtained energies. Since these offsets are of the same order as the energy gain arising from in-plane displacement of the atoms, this residual strain leads to a significant noise in the signal of the energy profile as a function of the imposed angle. To overcome this problem and to obtain a clear signal from our simulations, we enhanced the LJ-coupling strength between the $\mathrm{MoS}_{2}$ and $\mathrm{G}$ layers. In other words, we set the LJ-parameters $\epsilon_{i j}$ in Eq. (2) to $\epsilon_{i j}^{\prime}=100 \cdot \epsilon_{i j}$ during the geometry optimization. Next, the applied bias is corrected by scaling back the energy 

problem.

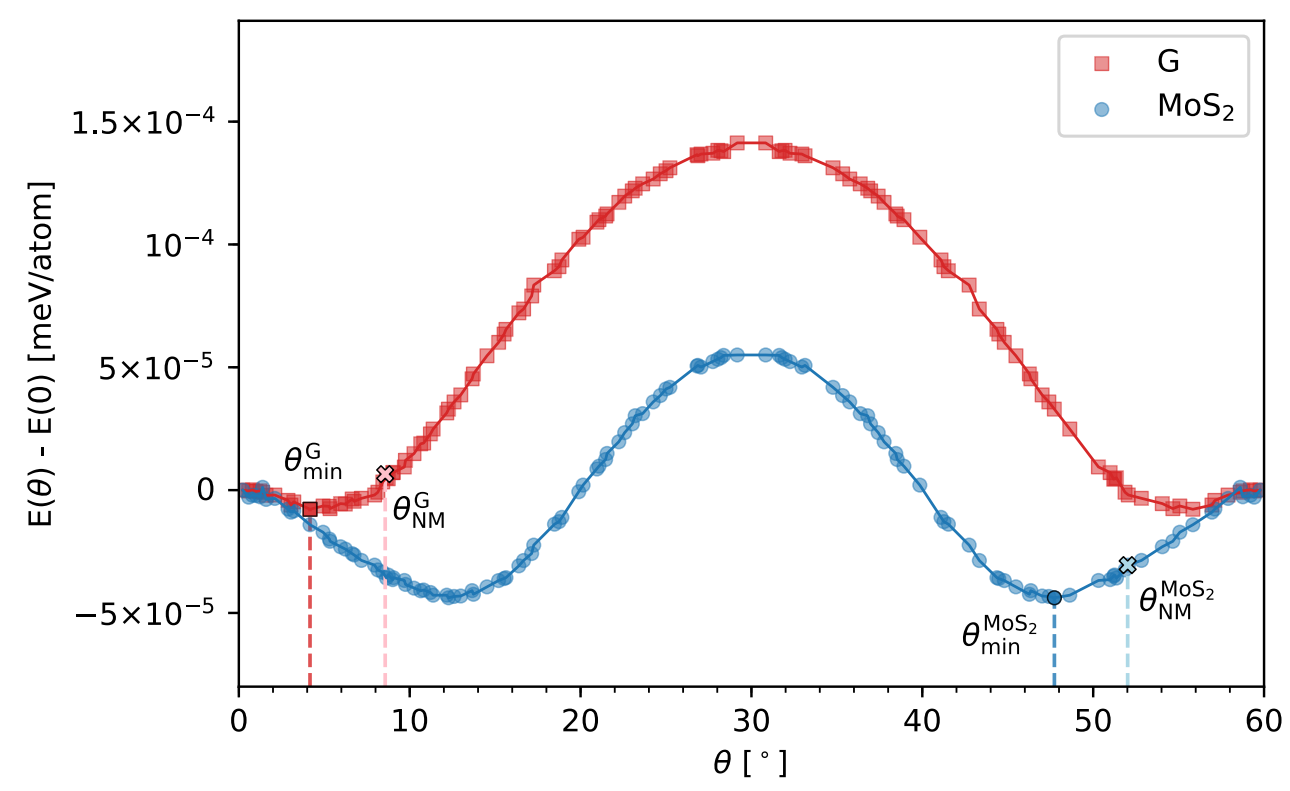

Figure 2: Energy $\mathrm{E}\left(\mathrm{meV} /\right.$ atom) as a function of the imposed angle $\theta\left(^{\circ}\right)$ for different $2 \mathrm{D}$ models: red squares refer to flexible $\mathrm{G}$ on top of rigid $\mathrm{MoS}_{2}$; blue circles refer to flexible $\mathrm{MoS}_{2}$ on top of rigid G. The labels in the legend indicate the flexible ML. The reference value of the energy scale is set to $E(0)$. The minimum-energy point along each curve is highlighted by a dashed line and a label. Red and blue crosses (accompanied by a label) mark the minimum angle predicted by the NM theory for the first and second case, respectively. The minimum-energy and NM-predicted angles for flexible $\mathrm{MoS}_{2}$ are reported starting from $60^{\circ}$ for easiness of reading.

profile, as if simulated with the original value of $\epsilon_{i j}$. As is shown in Section three of the SI, this computational trick solely reduces the noise without affecting the actual physics of the

Figure 2 shows the optimized energy $\mathrm{E}\left(\mathrm{meV} /\right.$ atom) as a function of the angle $\theta\left(^{\circ}\right)$, with respect to the energy of the aligned structures, $E(0)$. The two curves refer to the following models: 2D-adsorbed $\mathrm{G}$ atop rigid $\mathrm{MoS}_{2}$ (red) and 2D-adsorbed $\mathrm{MoS}_{2}$ atop rigid $\mathrm{G}$ (blue). Both cases reveal a minimum at a non-zero angle: for the adsorbed $\mathrm{G}$ case, the minimum is found at $\theta_{\min }^{\mathrm{G}}=4.2^{\circ}$, while for the adsorbed $\mathrm{MoS}_{2}$ case it is at $\theta_{\min }^{\mathrm{MoS}_{2}}=12.3^{\circ}$. Due to the intrinsic hexagonal symmetry of our system, each curve has two symmetrical minima centered around $30^{\circ}$. The simulations show that the physics described by the approximation of Eq. (4) is still valid, i.e. a non-zero minimum angle is observed for both cases. However, 
the absolute values of the predicted and observed angles are not in agreement, yielding a discrepancy of $\theta_{\text {min }}^{\mathrm{G}}-\theta_{\mathrm{NM}}^{\mathrm{G}}=-4.4^{\circ}$ in the case of $2 \mathrm{D}$-adsorbed $\mathrm{G}$, and $\theta_{\min }^{\mathrm{MoS}_{2}}-\theta_{\mathrm{NM}}^{\mathrm{MoS}_{2}}=4.3^{\circ}$, in the case of 2D-adsorbed $\mathrm{MoS}_{2}$.

A previous study of $\mathrm{G}$ and h-BN hetero-structures showed ${ }^{30}$ the NM model quantitatively describes the relaxation of the constrained system of these purely 2D materials. Here, the NM theory captures the basics of the physics, but is not able to describe satisfactorily the complex geometry of the bilayer system, especially in case of the flexible $\mathrm{MoS}_{2}$ layer. We attribute the poor prediction of the theory in our case to the internal 3D structure of the $\mathrm{MoS}_{2}$ monolayer, which indeed is unaccounted for in the NM model. This suggests that the NM theory is generally of limited utility for any bilayer comprising TMDs or other systems with a 3D monolayer structure. Another difference between our results and the results found for $\mathrm{G} / \mathrm{hBN}$ heterostructures ${ }^{300}$ is the quantitative difference in the energy values. In fact, our results report energy differences of one order of magnitude less. We explain this difference by the earlier mentioned relatively large $\mathrm{MoS}_{2} / \mathrm{G}$ BL system mismatch ratio $\rho=l / l \approx 0.8$. Due to this significant incommensurability between the $\mathrm{MoS}_{2}$ and G, present for all rotations, the atom displacements and energy differences are expected to be less pronounced. In fact, a similar observation has been done experimentally by Diaz et al. ${ }^{[27}$ and Pierucci et al. ${ }^{28}$ For example, Diaz et al. found that the $\mathrm{MoS}_{2} / \mathrm{G}$ moiré pattern obtain via AFM measurements was less pronounced compared to the one of $\mathrm{hBN} / \mathrm{G}$.

Considering another case, one in which all degrees of freedom are considered, i.e. all atoms are free to move in the 3D space, the NM theory is even qualitatively inadequate. Figure 3 shows the energy $\mathrm{E}\left(\mathrm{meV} /\right.$ atom) as a function of the angle $\theta\left(^{\circ}\right)$ of the system without rigid substrate, but with two soft, interacting layers. Naturally, the LJ-coupling between the two layers has been restored to the values obtained from fitting against the DFT data to correctly reproduce interlayer forces. The behaviour that we found is both quantitatively and qualitatively different from the constrained system presented previously. The introduction of the out-of-plane dimension $(z)$ changes the response qualitatively. The 


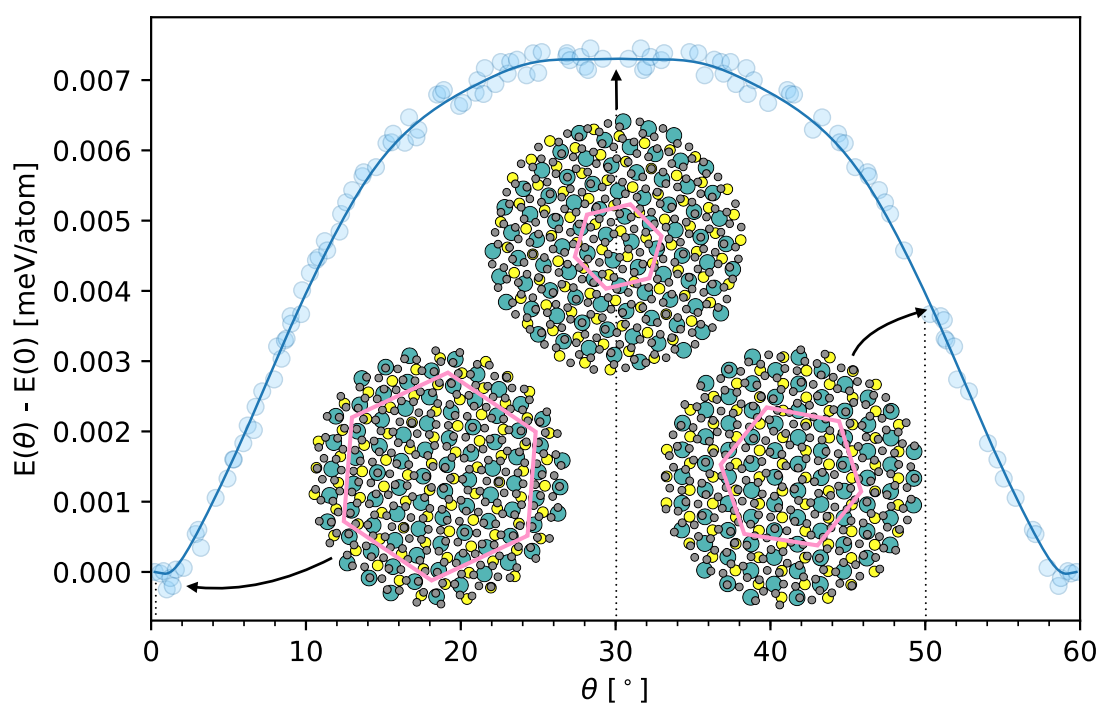

Figure 3: Energy per atom $E(\theta)$, in meV, as a function of the imposed angle $\theta$. Each point in the energy landscape represents a distinct geometry at a different imposed angle and the blue line is a Bézier fit. The small oscillations at $\theta=0^{\circ}, 60^{\circ}$ are due to numerical noise in the energy simulations. The circular insets show top views of the relaxed structures at $\theta \approx 0^{\circ}$ (bottom left), $\theta \approx 50^{\circ} \equiv 10^{\circ}$ (bottom right) and $\theta \approx 30^{\circ}$ (top center). The atoms are colored according to the scheme in Figure 1. The pink hexagons sketch the moiré tile in each configuration.

energy minima at non-zero angles have disappeared and the energy now rises symmetrically from the global minima at the aligned cases $\left(0^{\circ}, 60^{\circ}\right)$ towards the global maximum at the mismatch angle of $30^{\circ}$. From Figure 3, one can thus deduce that at $0 \mathrm{~K}$, the fully flexible bilayer system will be stable when aligned at $0^{\circ}$ or $60^{\circ}$. The energy profile around the misalignment of $30^{\circ}$ is flat and the misaligned geometry at this angle could therefore be characterized as meta-stable, as a vanishing force $F=-\mathrm{d} E(\theta) / \mathrm{d} \theta$ drives the system toward the global minima at $0^{\circ}$ or $60^{\circ}$. In the thermodynamic limit this orientation should not occur, considering it is the global energy maximum. The fact that non-aligned structures are observed experimentally ${ }^{23}$ can be explained by the fact that this type of geometry can be temporarily stabilized by a small internal friction, e.g. due to local defects, creating energy barriers that need to be overcome first.

The NM theory does not hold when structural distortions perpendicular to the interface are allowed. Our results indicate that these are important for $\mathrm{MoS}_{2} / \mathrm{G}$ heterostructures and 
we believe that this is also the case for other complex heterostructures. The core of the NM argument is that the collective misalignment arises due to the excitation of the transverse phonon branch in the $x y$ plane, which lies lower in energy than the longitudinal branch. This static distortion, which results in an increased internal energy of the adsorbate layer, is counterbalanced by a better interdigitation of the two lattices, that is, the displaced atoms are locally displaced to an overall more favorable stacking, with respect to the interlayer potential. ${ }^{4142}$ If out-of-plane distortions, unaccounted for in the NM theory, lead to a better interplay between the two layers, i.e. if there is a gain in the interlayer coupling energy that is larger than the intralayer energy penalty from out-of-plane modulations, the system will lower its total energy. Differently from the NM theory, the lowest-energy distortion in this scenario would not result in a misalignment between the components, but in the formation of ripples creating locally commensurate zones at the equilibrium distance and incommensurate zones at a larger interlayer distance.

We characterize here the out-of-plane deformations, disregarded in NM theory, in terms of the flexural phonon branch, which describes excitations perpendicular to the ML plane. In line with our edge-free geometries, the phonon picture is independent of sample size and, since the phonon eigenvectors represent a complete basis set, $\stackrel{45}{4}$ any distortion in the crystal may be expressed as a linear combination of phonons. Moreover, treating the distortions of a layer in terms of its phonon spectrum decouples the intralayer energetics from interlayer interaction. Our analysis, reported in Section seven of SI, shows that deformations in the $\mathrm{MoS}_{2} \mathrm{ML}$ are two orders of magnitude smaller than the deformation of G. This signals that the distortion governing the energy economy of the system happens in the G layer and we therefore focus the following analysis on this part only. As shown by the blue solid line in Figure 4, the flexural band is flat near the center of the Brillouin Zone (BZ) ( $\Gamma$ point), i.e. the long-wave modulations perpendicular to the basal plane can occur essentially without an energy penalty. Transverse (dashed grey in Figure 4) and longitudinal (dash-dotted gray in Figure (4) bands, that are the basis of NM theory, are higher in energy, resulting in more 
expensive modulations of the $\mathrm{G}$ layer.

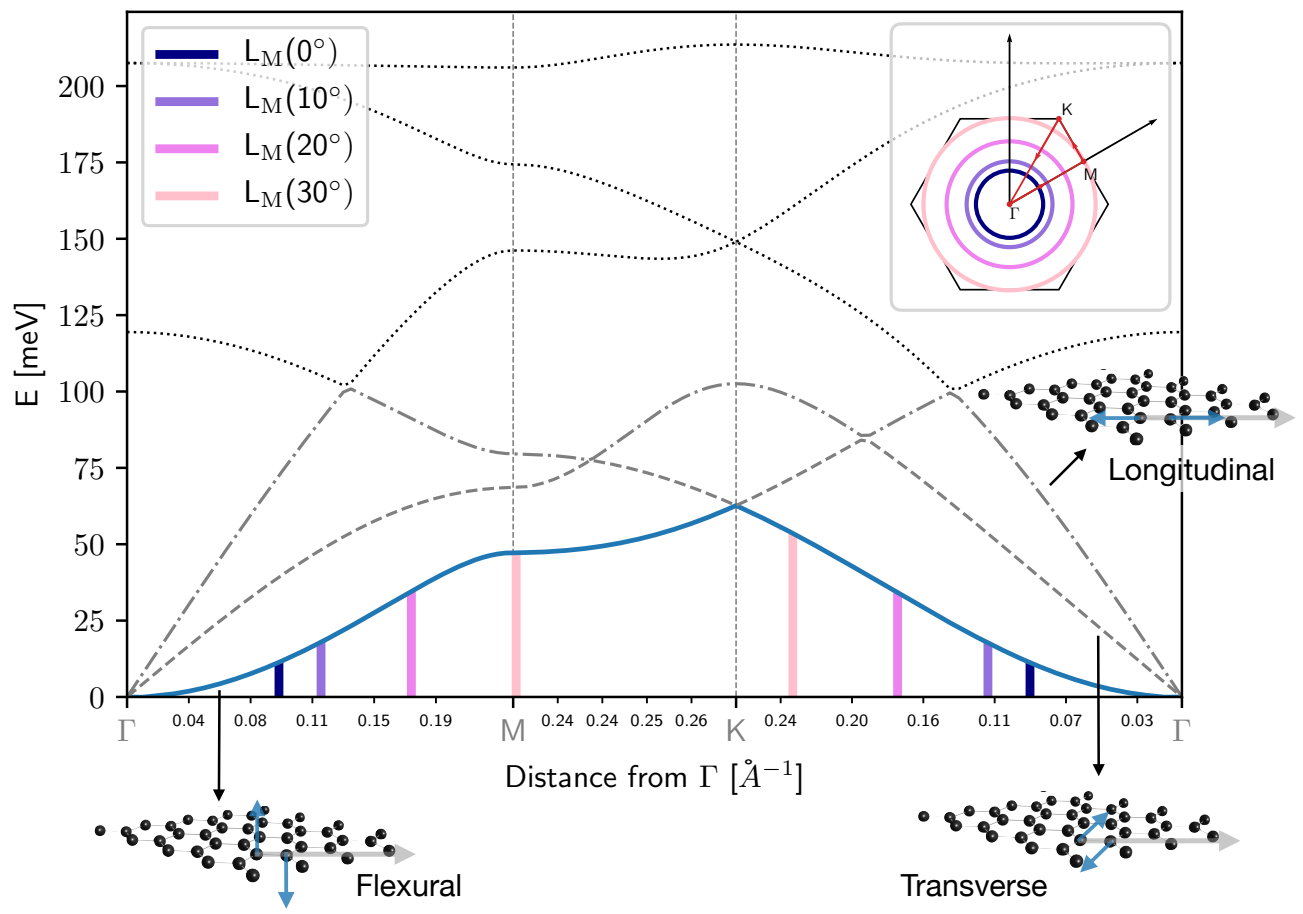

Figure 4: Phonon band structure of the $\mathrm{G}$ monolayer. The $y$ axis reports the phonon energy, while the $x$ axis marks the distance from the origin along the path $\Gamma \rightarrow M \rightarrow K \rightarrow \Gamma$, shown in the top right inset and marked along the $x$ axis by gray dashed lines. The flexural branch is reported by a solid blue line, transverse branch by dashed gray line and longitudinal branch by dash-dotted grey line while other branches are shown in dotted black lines. Colored segments along $x$ raising from $y=0$ to the flexural branch mark wavevectors matching the moiré spacing $L_{\mathrm{M}}(\theta)$ for the geometries in the insets of Figure 5, as highlighted by the color-code. The moiré wavevector $k_{\mathrm{M}}$ corresponding to real-space wavelength $L_{\mathrm{M}}$ are shown in the top-right inset following the color-code in the legend in top left. The insets marked by black arrows show the characteristic displacement pattern for the three acoustic branches: gray arrow indicates the direction of the wave vector $\vec{k}$ and blue arrow the displacement of the atoms in a unit cell.

While the phonon spectrum describes the energy penalty compared to an isolated ML at equilibrium, the energy gain from interlayer interactions can be quantified in terms of moiré patterns. The moiré superlattice is a geometrical construction describing the interference between two lattices, and can be used to identify geometrical correspondence between lattices, i.e. zones of local commensuration versus incoherent stacking. The symmetry of the moiré superlattice reflects the one underlying Bravais lattices and its lattice parameter $L_{\mathrm{M}}$ 
is given by

$$
L_{\mathrm{M}}(\theta)=\frac{l_{\mathrm{G}}}{\sqrt{1+\rho^{-2}-2 \rho^{-1} \cos \theta}} .
$$

Figure 5 shows the correlation between out-of-plane modulations, i.e. rippling in the $z$ dimension, and the moiré pattern in the $\mathrm{G}$ sheet. At $\theta=0$, the moiré spacing and the average displacement along $z$ are at a maximum and they both decrease as the misalignment increases. As $\theta$ increases, the length of the pattern shrinks with the displacement along $z$ : at $\theta=30^{\circ}$ the moiré shrinks to a couple of unit cells and the monolayer remains basically flat. The rippling patterns follow perfectly the moiré superlattice, as shown in the insets of Figure 5 for selected values of $\theta$, i.e. the nearest configurations having approximately an angle of $\theta=0^{\circ}\left(0.23^{\circ}\right), 10^{\circ}\left(9.75^{\circ}\right), 20^{\circ}\left(19.89^{\circ}\right), 30^{\circ}\left(29.17^{\circ}\right)$. The local information expressed in the ripples of the carbon sheet is condensed in the thickness of the G layer

$$
\tau(\theta)=\max _{\vec{r}_{i} \in \mathrm{G}} z_{i}-\min _{\vec{r}_{i} \in \mathrm{G}} z_{i},
$$

which is a global parameter with a single value for each twisted geometry, as reported by black circles on the left $y$ axis in Figure 5 .

Combining the geometrical construction shown in Figure 5 and the $\mathrm{G}$ phonon dispersion in Figure 4, we can explain the energy profile in Figure 3. The moiré spacing $L_{\mathrm{M}}(\theta)$ can be mapped to wavevectors into the BZ by $k_{\mathrm{M}}(\theta)=\frac{2}{\sqrt{3} L_{\mathrm{M}}(\theta)}$. The wavevectors $k_{\mathrm{M}}$, corresponding to the spacing $L_{\mathrm{M}}$ of the geometries in the insets of Figure 5, are highlighted in Figure 4. All wave-vectors in the BZ at the moiré spacing are shown by vertical segments along the path and as circles in the inset, and follow the same color-code. A modulation of the G sheet, with wavelength $L_{\mathrm{M}}(\theta)$, can be represented as a combination of phonon modes of matching wavevectors $k_{\mathrm{M}}(\theta)$. Since the modulation of $\mathrm{G}$ layer essentially takes solely place in the $z$ direction, the major contribution in the decomposition on phonon modes will come from the flexural branch. Within small displacements from the equilibrium positions, the energy price of such modulations can be estimated by the corresponding phonon energy. As $\theta$ varies from 


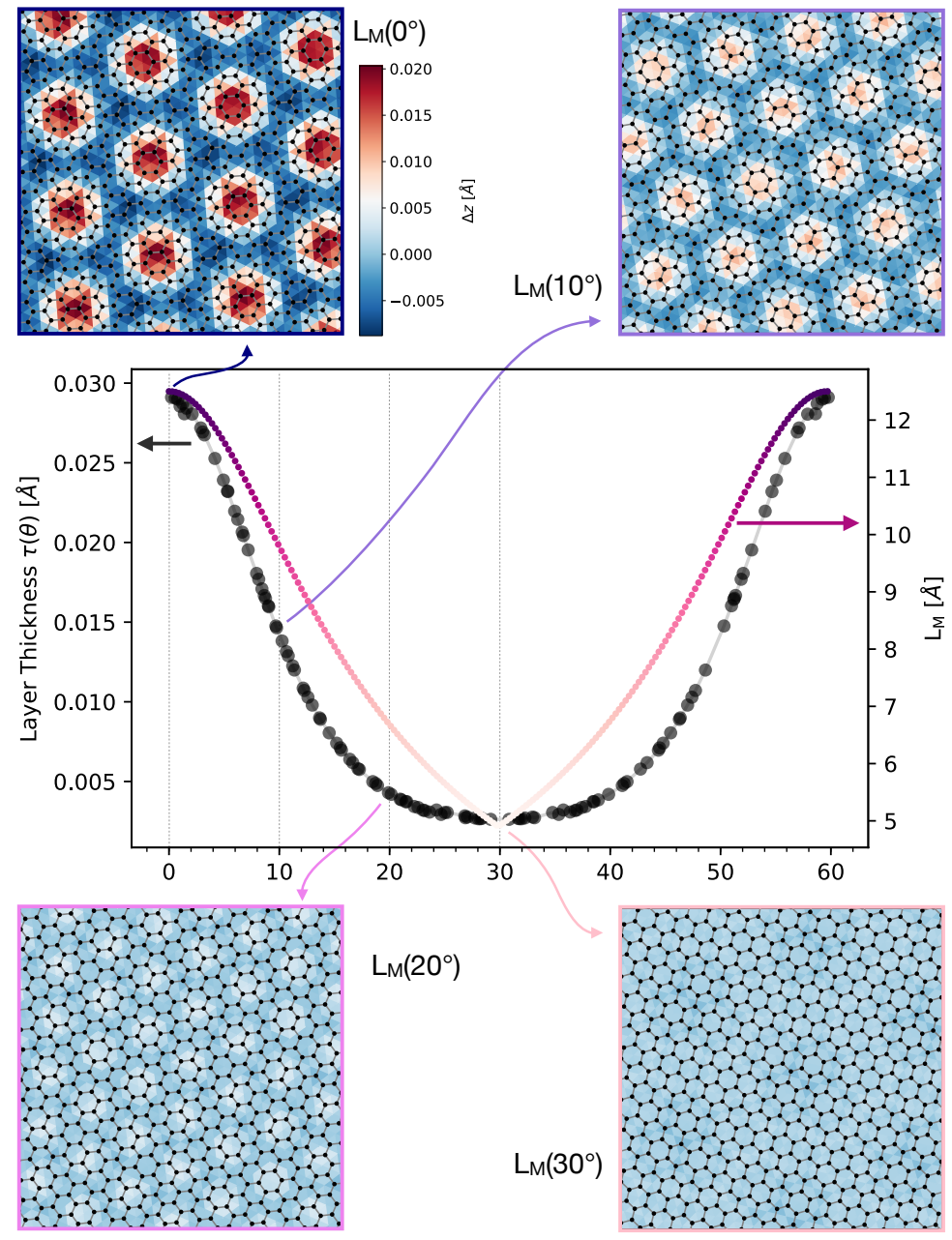

Figure 5: Thickness of $\mathrm{G}$ layer $\tau(\theta)$ resulting from the displacement of $\mathrm{C}$ atoms (black circles, left axis) and spacing of the moiré pattern $L_{\mathrm{M}}(\theta)$ (colored dotted line, right axis) as a function of $\theta$ for equivalent configurations at $0^{\circ}$ and $60^{\circ}$ rotating towards $30^{\circ}$. The insets show the local distortion following the moiré lattice in a square of sides $60 \AA$ at the nearest configuration having approximately an angle of $\theta=0^{\circ}\left(0.23^{\circ}\right.$, dark purple), $10^{\circ}\left(9.75^{\circ}\right.$, purple), $20^{\circ}\left(19.89^{\circ}\right.$, dark pink $), 30^{\circ}\left(29.17^{\circ}\right.$, pink $)$. The color of each triangle reports change in height $\Delta z=z-z_{\text {eq }}$ coordinate of the corresponding $\mathrm{C}$ atom (black points) following the color code reported in top left. For example, the moiré pattern can be seen in the inset for $\mathrm{L}_{\mathrm{M}}\left(0^{\circ}\right)$ as the lattice defined by the red regions. 
$0^{\circ}$ to $30^{\circ}$ and the moiré shrinks, like is shown in Figure 5, the associated wavevector $k_{\mathrm{M}}(\theta)$ assumes the values between $k_{M}\left(0^{\circ}\right)=0.09 \AA^{-1}$ (dark-purple lines in Figure 4) and $k_{M}\left(30^{\circ}\right)=$ $0.24 \AA^{-1}$ (pink lines in Figure 4), at increasing flexural-phonon energies. Next, we describe these limiting cases in more detail. At $\theta=0^{\circ}$, the spacing of the moiré is $L_{\mathrm{M}}=12.5 \AA$, which is the distance between the locally commensurate patches, the red regions in Figure 5 . As signaled by the dark-purple line in Figure 4, flexural phonon modes of this length in $\mathrm{G}$ are close to the flat region around $\Gamma$ and therefore energetically inexpensive. This allows commensurate regions to stay at the equilibrium interlayer position, while incommensurate ones are pushed away from $\mathrm{MoS}_{2} \mathrm{ML}$, perpendicular to the basal planes. As $\theta$ increases to $30^{\circ}, L_{\mathrm{M}}$ decreases, and thus the distance between locally commensurate areas reduces. As a result, the deformation needs to occur over a shorter distance and its energy cost therefore increases. At $\theta=30^{\circ}, L_{M}=4.88 \AA$, which is about $2 \mathrm{G}$ unit cells. As shown by the pink line in Figure 4, deformations of this length scale are described by phonons at the edges of the BZ and are energetically more expensive than the gain coming from the interdigitation with the substrate. Therefore the $\mathrm{G}$ sheet remains flat, at the expense of the interlayer coupling, resulting in a higher total energy of the heterostructure compared to the aligned case. The flattening of the flexural branch near the edges of the BZ, as shown by the solid blue line between $\mathrm{M}$ and $\mathrm{K}$ in Figure 4 , is mirrored by the plateau in the energy profile in Figure 3 around $\theta=30^{\circ}$ : in the whole region moiré modulations are too expensive and the system cannot obtain any energy gain.

To sum up, the unconstrained 3D heterostructure lowers its energy by out-of-plane distortions according to the moiré pattern. This is particularly evident at $\theta=0$, where $L_{\mathrm{M}}=12.5 \AA$ : here the flexural distortion is almost without any energy penalty and the system lowers its energy by improving the interdigitation between $\mathrm{G}$ and the $\mathrm{MoS}_{2}$ layers. As $\theta$ increases, $L_{\mathrm{M}}$ decreases and the cost of the ripples overtakes the gain in energy due to local commensuration, yielding flat $\mathrm{G}$ and an increased total energy. Finally, the region of the BZ spanned by the moiré spacing as a function of $\theta$ (the region between purple and 
pink segments in Figure 44 shows that the approximation of long-wavelength used to derive Eq. (4) is unsuited in the large-mismatched heterostructures. Because of the fact that also phonon excitation near the border of the BZ are involved in the energy economy of the system, a modified epitaxy theory is needed in these cases to predict and to understand the phase stability of imposed twist angles.

\section{Conclusions}

We explored the stability of twisted vdW heterostructures. Although often overlooked, this phenomenon is of particular importance in the emerging field of twistronics, as it can be a decisive factor in the real-life application of such systems. The energy as a function of an imposed angle determines whether a device is at risk of rotating away from a prepared angle. Our analysis of $\mathrm{MoS}_{2} / \mathrm{G}$ heterostructures helps to clarify the scattered experimental data. We find a single global minimum at $\theta=0^{\circ}$ and $60^{\circ}$ : i.e. only epitaxial stacking is expected for the system at $0 \mathrm{~K}$. However, experiments always present defects or intrinsic friction that might result in the emergence of activation energies, potentially trapping a system in a meta-stable (or even unstable) state. In the limit where such effects become negligible, i.e. activation energy approaching zero, one would mostly observe aligned at $\theta=0^{\circ}$ and $60^{\circ}$ and few $30^{\circ}$-rotated heterostructures, in agreement with the results of Liu et al. ${ }^{23} \mathrm{~A}$ possible experiment, to test the validity of our results, would be to perform a systematic repetition of the aforementioned experiments, focused upon reducing deviations resulting from working conditions, e.g. annealing temperature. We expect that the results of such an effort will confirm our findings: with a high enough annealing temperature and large enough flakes of significant quality, the bilayer system should be found in the aligned configuration, with possibly some outliers around $30^{\circ}$.

The relevance of our results becomes clear when considering the fact that interesting physics is observed at certain unstable mismatch angles. ${ }^{28}$ Our findings show that care must 
be taken when designing nanodevices as properties observed in studies at a specific angle might fade over time due to the system spontaneously rotating toward the real thermodynamic equilibrium. In fact, others $\frac{19}{19}$ also suggest that aligned structures are most suitable for optospintronic applications.

Finally, we explain the origin of the observed energy economy in terms of the interplay between flexural phonon modes of the pristine compounds and the moiré superlattice. This insight is general in nature and can be applied to all layered materials and heterostructures, serving as a design tool for twistronic devices. Stiffness in the out-of-plane direction should be considered as a critical property in the design of such devices. It is for example known that rippling can affect the Schottky junctions, which are directly related to the performance of optoelectronic devices such as photodetectors and solar cells. ${ }^{477}$ Soft flexural phonon modes might be a lower energy route out of frustration than twisting, hindering the possibility of stable rotated configurations. Furthermore, our results show the need for a novel theory of epitaxy for layered materials, incorporating the flexural branches ignored in the NM theory and taking into account all phonon wavelengths. The insights presented here can serve as a starting point for developing such a theory of the epitaxial growth for vdW heterostructures.

\section{Methods}

Classical MD All energy minimizations of the rotated heterostructures have been performed using molecular dynamics by means of the LAMMPS package ${ }^{40}$ using the conjugate gradient algorithm, where the energy tolerance was set to $1 \times 10^{-15}$. The REBO potential ${ }^{34}$ was used for G, whereas an adapted version of the 3-body Stillinger-Weber (SW) potential ${ }^{\sqrt{33}}$ was used for $\mathrm{MoS}_{2}$. To model the vdW interactions, we used an interlayer LJ potential. To obtain the explicit values of the parameters, we refined the values that can be found in the paper by Ding et al.,, 33 of which we provide an elaborate description in the SI. 
DFT calculations Ab initio calculations used to re-parametrise the force field were carried out using DFT as implemented in the Vienna Ab initio Simulation Package (VASP) within the Projector Augmented-Wave (PAW) framework. ${ }^{37}$ The exchange-correlation potential is approximated using the PBE functional ${ }^{38}$ and the vdW dispersion is described by DFT-D2 method. ${ }^{39}$ A plane wave cut-off of $800 \mathrm{eV}$ is adopted and the Brillouin zone was sampled using a $13 \times 13 \times 1$ mesh.

Phonon calculation Phonon bands were computed with the aid of Phonopy, was coupled to LAMMPS using phonoLAMMPS. ${ }^{49}$ In both cases the phonon dispersion was computed using the frozen method employing a 5x5x1 supercell.

\section{Acknowledgement}

The authors are thankful to E. Tosatti, A. Vanossi, D. Mandelli and M. Liao for the helpful discussions. This project has received funding from the European Union's Horizon2020 research and innovation programme under grant agreement No. 721642: SOLUTION. The authors acknowledge the use of the IRIDIS High Performance Computing Facility, and associated support services at the University of Southampton, in the completion of this work. This work was also supported by The Ministry of Education, Youth and Sports from the Large Infrastructures for Research, Experimental Development and Innovations project "IT4Innovations National Supercomputing Center - LM2015070" and by the project Novel nanostructures for engineering applications No. CZ.02.1.01/0.0/0.0/16_026/0008396.

\section{Additional information}

Author Contributions AS and VEPC carried out all calculations and data analysis and conceptualize the study in the first place. PN, DK supervised extensively the study and TP guided the project. All authors contributed to the writing of this work. Finally, PN, DK 
and TP secured funding acquisition.

Supporting Information Details on the protocols, force field and geometries parameters are supplied as Supporting Information (DOI here).

Competing interests The authors declare no competing interests.

\section{References}

(1) Mannix, A. J.; Kiraly, B.; Hersam, M. C.; Guisinger, N. P. Synthesis and Chemistry of Elemental 2D Materials. Nature Reviews Chemistry 2017, 1, 0014.

(2) Chhowalla, M.; Shin, H. S.; Eda, G.; Li, L.-J.; Loh, K. P.; Zhang, H. The Chemistry of Two-Dimensional Layered Transition Metal Dichalcogenide Nanosheets. Nature Chemistry 2013, 5, 263-275.

(3) Vazirisereshk, M. R.; Martini, A.; Strubbe, D. A.; Baykara, M. Z. Solid Lubrication with $\mathrm{MoS}_{2}$ : A Review. Lubricants 2019, 7, 57.

(4) Radisavljevic, B.; Radenovic, A.; Brivio, J.; Giacometti, V.; Kis, A. Single-Layer MoS 2 Transistors. Nature Nanotechnology 2011, 6, 147-150.

(5) Lopez-Sanchez, O.; Lembke, D.; Kayci, M.; Radenovic, A.; Kis, A. Ultrasensitive Photodetectors Based on Monolayer $\mathrm{MoS}_{2}$. Nature Nanotechnology 2013, 8, 497-501.

(6) Lauritsen, J. V.; Bollinger, M. V.; Lægsgaard, E.; Jacobsen, K. W.; Nørskov, J. K.; Clausen, B. S.; Topsøe, H.; Besenbacher, F. Atomic-Scale Insight into Structure and Morphology Changes of $\mathrm{MoS}_{2}$ Nanoclusters in Hydrotreating Catalysts. Journal of Catalysis 2004, 221, 510-522.

(7) Shi, X.-R.; Jiao, H.; Hermann, K.; Wang, J. CO Hydrogenation Reaction on Sulfided Molybdenum Catalysts. Journal of Molecular Catalysis A: Chemical 2009, 312, 7-17. 
(8) Shi, X.-R.; Wang, S.-G.; Hu, J.; Wang, H.; Chen, Y.-Y.; Qin, Z.; Wang, J. Density Functional Theory Study on Water-Gas-Shift Reaction over Molybdenum Disulfide. Applied Catalysis A: General 2009, 365, 62-70.

(9) Dienwiebel, M.; Verhoeven, G. S.; Pradeep, N.; Frenken, J. W. M.; Heimberg, J. A.; Zandbergen, H. W. Superlubricity of Graphite. Physical Review Letters 2004, 92, 126101.

(10) Vazirisereshk, M. R.; Ye, H.; Ye, Z.; Otero-de-la Roza, A.; Zhao, M.-Q.; Gao, Z.; Johnson, A. T. C.; Johnson, E. R.; Carpick, R. W.; Martini, A. Origin of Nanoscale Friction Contrast between Supported Graphene, $\mathrm{MoS}_{2}$, and a Graphene/MoS $\mathrm{M}_{2}$ Heterostructure . Nano Letters 2019, 19, 5496-5505.

(11) Geim, A. K.; Grigorieva, I. V. Van der Waals Heterostructures. Nature 2013, 499, $419-425$.

(12) Novoselov, K. S.; Mishchenko, A.; Carvalho, A.; Castro Neto, A. H. 2D Materials and van der Waals Heterostructures. Science 2016, 353, 9439.

(13) Liu, Y.; Weiss, N. O.; Duan, X.; Cheng, H.-C.; Huang, Y.; Duan, X. Van der Waals Heterostructures and Devices. Nature Reviews Materials 2016, 1, 16042.

(14) Zhang, K.; Fang, X.; Wang, Y.; Wan, Y.; Song, Q.; Zhai, W.; Li, Y.; Ran, G.; Ye, Y.; Dai, L. Ultrasensitive Near-Infrared Photodetectors Based on a Graphene$\mathrm{MoTe}_{2}$-Graphene Vertical van der Waals Heterostructure. ACS Applied Materials and Interfaces 2017, 9, 5392-5398.

(15) Zhu, S.; Pochet, P.; Johnson, H. T. Controlling Rotation of Two-Dimensional Material Flakes. ACS Nano 2019, 13, 6925-6931.

(16) Du, L.; Yu, H.; Liao, M.; Wang, S.; Xie, L.; Lu, X.; Zhu, J.; Li, N.; Shen, C.; Chen, P.; Yang, R.; Shi, D.; Zhang, G. Modulating PL and Electronic Structures of 
$\mathrm{MoS}_{2} /$ Graphene Heterostructures via Interlayer Twisting Angle. Applied Physics Letters 2017, 111, 263106.

(17) Huang, Y. L.; Chen, Y.; Zhang, W.; Quek, S. Y.; Chen, C.-H.; Li, L.-J.; Hsu, W.-T.; Chang, W.-H.; Zheng, Y. J.; Chen, W.; Wee, A. T. S. Bandgap Tunability at SingleLayer Molybdenum Disulphide Grain Boundaries. Nature Communications 2015, 6, 6298.

(18) Martin, J. M.; Erdemir, A. Superlubricity: Friction's Vanishing Act. Physics Today 2018, $71,40-46$.

(19) Du, L.; Zhang, Q.; Gong, B.; Liao, M.; Zhu, J.; Yu, H.; He, R.; Liu, K.; Yang, R.; Shi, D.; Gu, L.; Yan, F.; Zhang, G.; Zhang, Q. Robust spin-valley polarization in commensurate $\mathrm{MoS}_{2}$ /graphene heterostructures. Physical Review B 2018, 97, 115445.

(20) Zan, R.; Ramasse, Q.; Jalil, R.; Tu, J.-S.; Bangert, U.; Novoselov, K. Imaging Two Dimensional Materials and their Heterostructures. J. Phys.: Conf. Ser. 2017, 902, 012028.

(21) Cao, Y.; Fatemi, V.; Fang, S.; Watanabe, K.; Taniguchi, T.; Kaxiras, E.; JarilloHerrero, P. Unconventional Superconductivity in Magic-Angle Graphene Superlattices. Nature 2018, 556, 43-50.

(22) Liao, M.; Wu, Z.-W.; Du, L.; Zhang, T.; Wei, Z.; Zhu, J.; Yu, H.; Tang, J.; Gu, L.; Xing, Y.; Yang, R.; Shi, D.; Yao, Y.; Zhang, G. Twist Angle-Dependent Conductivities Across $\mathrm{MoS}_{2}$ /Graphene Heterojunctions. Nature Communications 2018, 9, 4068.

(23) Liu, X.; Balla, I.; Bergeron, H.; Campbell, G. P.; Bedzyk, M. J.; Hersam, M. C. Rotationally Commensurate Growth of $\mathrm{MoS}_{2}$ on Epitaxial Graphene. ACS Nano 2016, 10, 1067-1075. 
(24) Shi, Y.; Zhou, W.; Lu, A.-Y.; Fang, W.; Lee, Y.-H.; Hsu, A. L.; Kim, S. M.; Kim, K. K.; Yang, H. Y.; Li, L.-J.; Idrobo, J.-C.; Kong, J. Van der Waals Epitaxy of MoS $\mathrm{M}_{2}$ Layers Using Graphene As Growth Templates. Nano Letters 2012, 12, 2784-2791.

(25) Lu, C. I.; Butler, C. J.; Huang, J. K.; Hsing, C. R.; Yang, H. H.; Chu, Y. H.; Luo, C. H.; Sun, Y. C.; Hsu, S. H.; Yang, K. H. O.; Wei, C. M.; Li, L. J.; Lin, M. T. Graphite Edge Controlled Registration of Monolayer $\mathrm{MoS}_{2}$ Crystal Orientation. Applied Physics Letters 2015, 106, 2-6.

(26) Adrian, M.; Senftleben, A.; Morgenstern, S.; Baumert, T. Complete Analysis of a Transmission Electron Diffraction Pattern of a $\mathrm{MoS}_{2}$-Graphite Heterostructure. Ultramicroscopy 2016, 166, 9-15.

(27) Diaz†, H. C.; Avila, J.; Chen, C.; Addou, R.; Asensio, M. C.; Batzill, M. Direct Observation of Interlayer Hybridization and Dirac Relativistic Carriers in Graphene/MoS 2 van der Waals Heterostructures. Nano Letters 2015, 15, 1135-1140.

(28) Pierucci, D.; Henck, H.; Avila, J.; Balan, A.; Naylor, C. H.; Patriarche, G.; Dappe, Y. J.; Silly, M. G.; Sirotti, F.; Johnson, A. T. C.; Asensio, M. C.; Ouerghi, A. Band Alignment and Minigaps in Monolayer $\mathrm{MoS}_{2}$-Graphene van der Waals Heterostructures. Nano Letters 2016, 16, 4054-4061.

(29) Naik, M. H.; Maity, I.; Maiti, P. K.; Jain, M. Kolmogorov-Crespi Potential for Multilayer Transition-Metal Dichalcogenides: Capturing Structural Transformations in Moiré Superlattices. Journal of Physical Chemistry C 2019, 123, 9770-9778.

(30) Guerra, R.; van Wijk, M.; Vanossi, A.; Fasolino, A.; Tosatti, E. Graphene on h-BN: to Align or Not to Align? Nanoscale 2017, 9, 8799-8804.

(31) Wang, Y.; Xiao, J.; Zhu, H.; Li, Y.; Alsaid, Y.; Fong, K. Y.; Zhou, Y.; Wang, S.; Shi, W.; Wang, Y.; Zettl, A.; Reed, E. J.; Zhang, X. Structural Phase Transition in Monolayer $\mathrm{MoTe}_{2}$ Driven by Electrostatic Doping. Nature 2017, 550, 487-491. 
(32) Wang, Z. J.; Ma, T. B.; Hu, Y. Z.; Xu, L.; Wang, H. Energy Dissipation of Atomicscale Friction Based on One-Dimensional Prandtl-Tomlinson Model. Friction 2015, 3, $170-182$.

(33) Ding, Z.; Pei, Q. X.; Jiang, J. W.; Huang, W.; Zhang, Y. W. Interfacial Thermal Conductance in Graphene/ $\mathrm{MoS}_{2}$ Heterostructures. Carbon 2016, 96, 888-896.

(34) Brenner, D. W.; Shenderova, O. A.; Harrison, J. A.; Stuart, S. J.; Ni, B.; Sinnott, S. B. A Second-Generation Reactive Empirical Bond Order (REBO) Potential Energy Expression for Hydrocarbons. Journal of Physics-Condensed Matter 2002, 14, 783-802.

(35) Kresse, G.; Hafner, J. Ab Initio Molecular Dynamics for Open-Shell Transition Metals. Physical Review B 1993, 48, 13115-13118.

(36) Kresse, G.; Joubert, D. From Ultrasoft Pseudopotentials to the Projector AugmentedWave Method. Physical Review B 1999, 59, 1758-1775.

(37) Blöchl, P. E. Projector Augmented-Wave Method. Physical Review B 1994, 50, 1795317979.

(38) Perdew, J. P.; Burke, K.; Ernzerhof, M. Generalized Gradient Approximation Made Simple. Physical Review Letters 1996, 777, 3865-3868.

(39) Grimme, S. Semiempirical GGA-type Density Functional Constructed with a LongRange Dispersion Correction. Journal of Computational Chemistry 2006, 27, 17871799.

(40) Plimpton, S. Fast Parallel Algorithms for Short-Range Molecular Dynamics. Journal of Computational Physics 1995, 117, 1-19.

(41) Novaco, A. D.; McTague, J. P. Orientational Epitaxy-The Orientational Ordering of Incommensurate Structures. Physical Review Letters 1977, 38, 1286-1289. 
(42) McTague, J. P.; Novaco, A. D. Substrate-Induced Strain and Orientational Ordering in Adsorbed Monolayers. Physical Review B 1979, 19, 5299-5306.

(43) Brazda, T.; Silva, A.; Manini, N.; Vanossi, A.; Guerra, R.; Tosatti, E.; Bechinger, C. Experimental Observation of the Aubry Transition in Two-Dimensional Colloidal Monolayers. Physical Review X 2018, 8, 011050.

(44) Panizon, E.; Guerra, R.; Tosatti, E. Ballistic Thermophoresis of Adsorbates on FreeStanding Graphene. Proceedings of the National Academy of Sciences 2017, 114, E7035-E7044.

(45) Wallace, D. C.; Callen, H. Thermodynamics of Crystals. American Journal of Physics 1972, 40, 1718-1719.

(46) Mandelli, D.; Vanossi, A.; Manini, N.; Tosatti, E. Friction Boosted by Equilibrium Misalignment of Incommensurate Two-Dimensional Colloid Monolayers. Physical Review Letters 2015, 114, 108302.

(47) Tomer, D.; Rajput, S.; Li, L. Spatial inhomogeneity in Schottky barrier height at graphene/ $\mathrm{MoS}_{2}$ Schottky junctions. J. Phys. D: Appl. Phys. 2017, 50, 165301.

(48) Togo, A.; Tanaka, I. First Principles Phonon Calculations in Materials Science. Scripta Materialia 2015, 108, 1-5.

(49) Carreras, A. phonoLAMMPS. 2019; https://github.com/abelcarreras/ phonolamms. 